\title{
The Development of High-Current Power Supply System for Electrolytic Copper Foil
}

\author{
An Luo*, Fujun $\mathrm{Ma}^{\dagger}$, Qiaopo Xiong ${ }^{* *}$, and Zhixing $\mathrm{He}^{*}$ \\ ${ }^{*}$ College of Electrical and Information Engineering, Hunan University, Changsha, China \\ ${ }^{* *}$ 722th Research Institute, China Shipbuilding Industry Corporation (CSIC), Wuhan, China
}

\begin{abstract}
A $6.5 \mathrm{~V} / 50 \mathrm{kA}$ high-frequency switching power supply (HSPS) system composed of 10 power modules is developed to meet the requirements of copper-foil electrolysis. The power module is composed of a two-leg pulse width modulation (PWM) rectifier and a DC/DC converter. The DC/DC converter adopts two full-wave rectifiers in parallel to enhance the output. For the two-leg PWM rectifier, the ripple of the DC-link voltage is derived. A composite control method with a ripple filter is then proposed to effectively improve the performance of the rectifier. To meet the process demand of copper-foil electrolysis, the virtual impedance-based current-sharing control method with load current full feedforward is proposed for n-parallel DC/DC converters. The roles of load current feedforward and virtual impedance are analyzed, and the current-sharing control model of the HSPS system is derived. Virtual impedance is used to adjust the current-sharing impedance without changing the equivalent output impedance, which can effectively reduce current-sharing errors. Finally, simulation and experimental results verify the structure and control method.
\end{abstract}

Key words: Composite control, Current sharing, Feedforward control, High frequency, Switching power supply, Two-leg rectifier

\section{INTRODUCTION}

Electronic copper foil is an important raw material in the electronic and electrical industries; it provides the primary substrate and conductive circuits in printed circuit boards [1]. The DC power supply is the key device in the production of electrolytic copper foil [2]. It must be low in voltage but high in current, with an output current that can be more than $50 \mathrm{kA}$. The performance of the power supply is directly related to the quality, cost, and efficiency of copper-foil production. This power supply traditionally adopts phase-controlled thyristor rectifiers, which are composed of thyristors, step-down transformer, and diode rectifier [3]. The power conversion of this power supply is conducted at fundamental frequency. Thus, this power supply has shortcomings, including high power loss, poor control accuracy, and harmonic pollution.

Manuscript received Jun. 23, 2014; accepted Dec. 3, 2014

Recommended for publication by Associate Editor Tae-Woong Kim.

${ }^{\dagger}$ Corresponding Author: mafujun2004@163.com

Tel: +86-0731-88823964, Fax: +86-0731-88823700, Hunan University

${ }^{*}$ College of Electrical and Information Eng., Hunan University, China

${ }^{* *} 722$ th Research Inst., China Shipbuilding Industry Corp. (CSIC), China
High-frequency switching power supplies (HSPSs) have thus been developed [4]-[7] in low-voltage electrolytic areas. By using high-frequency conversion technology, HSPS can realize efficient power conversion and improve output quality. Compared with the thyristor power supply, HSPS has advantages, including high efficiency, small size, and good flexibility. Nowadays, this power system is increasingly being used in the electrolytic industry.

HSPSs are generally composed of an AC/DC rectifier and a DC/DC converter. The diode uncontrolled rectifier is commonly used as the pre-rectifier to provide a stable DC source [7]. It can produce a certain amount of harmonic currents that can seriously pollute the power system. To improve the quality of HSPS and purify the power grid, power factor correction (PFC) technology is developed to achieve high efficiency and low harmonic rectification [8]-[10]. For the three-phase PFC, several structures are proposed [11]-[15]. The widely used three-phase three-leg topology [13] can continuously control input current by pulse width modulation (PWM). However, this rectifier topology has six power switches and is unsuitable for low- and medium-power applications. To reduce switch number and 


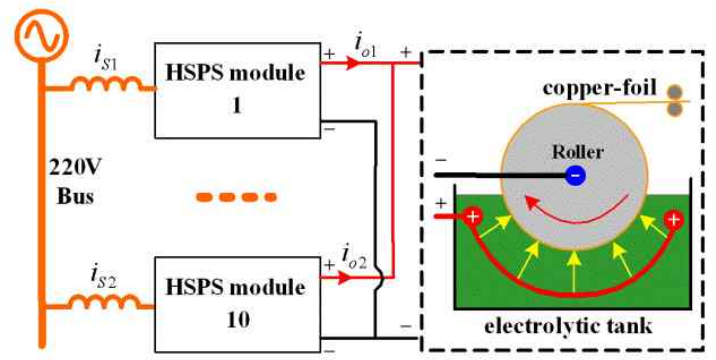

(a)

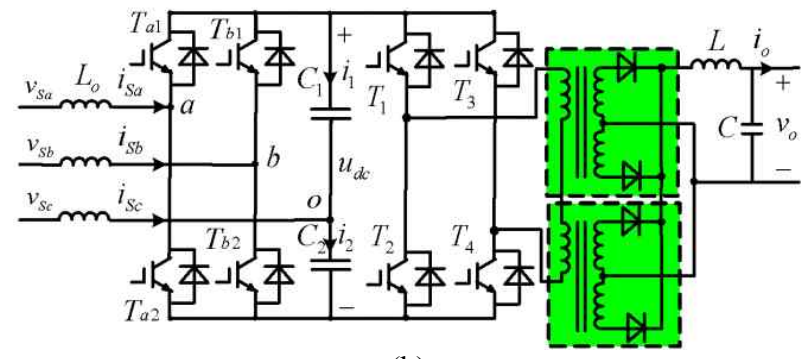

(b)

Fig. 1. Structure of the power supply for electrolytic copper foil. (a) Structure of the HSPS system. (b) Topology of the power module based on the PWM rectifier.

power loss, the three-phase two-leg topology is investigated and developed [14], [15]. However, two capacitors are present in the DC-link, and this structure has to control the voltage balance of these capacitors.

The back DC/DC converter of HSPS is composed of a single-phase full-bridge inverter, a diode rectifier, and an output filter. Copper-foil electrolysis is a dynamic engineering process with precision. The equivalent resistance of the load electrolytic solution is very small and easy to vary, which affects the stable operation of power supply. To improve dynamic performance of the DC/DC converter, the load current feedforward method is adopted to improve the anti-disturbance ability of load [16], [17]. Meanwhile, according to the process requirements of electrolytic copper foil, the power supply output is required to have a high current. Thus, the power supply system will be composed of multi-parallel DC/DC converters, and the critical current-sharing issue should be achieved to maintain a reliable and stable operation of the system. Dispersion of the main circuit parameter, nonlinear link, and mismatched regulator are consistently regarded as main factors causing output current-sharing errors. However, other factors that can cause output errors, such as random dead time and voltage loss of switches (diodes), are often overlooked [18]. To achieve the current-sharing control of a power supply system, a few methods were proposed, such as droop control, master/slave control, and average-current control [19]-[21].

A few droop control methods based on virtual impedance were recently proposed for the n-parallel AC inverters to eliminate the circulating current [17]-[18], [22], [23]. These methods generally realize virtual impedance through the inductive current feedback to enhance the equivalent output impedance of the converter, which can inhibit circulating currents. These methods can also be used for DC/DC converters to eliminate current-sharing errors. However, virtual impedance, which is used to increase the equivalent output impedance, can increase output voltage loss. The load harmonic currents can also easily distort output voltage.

In this paper, a $6.5 \mathrm{~V} / 50 \mathrm{kA}$ HSPS system for electrolytic copper foil is investigated. First, the topology of the HSPS system for copper-foil electrolysis is described in Section II. The model of the three-phase two-leg PWM rectifier is analyzed, and the AC ripple of the DC-link voltage is derived. A composite control method is then proposed for the PWM rectifier in Section III. For the control of DC/DC power modules, the virtual impedance-based current-sharing control method is proposed and its closed-loop control model is derived and analyzed in Section IV. Finally, simulation and experiment are conducted in Sections V and VI respectively, and the industrial application is presented to verify the proposed system.

\section{TOPOLOGY OF HSPS SYSTEM FOR ELECTROLYTIC COPPER-FOIL}

The diagram of copper-foil electrolysis is shown in Fig. 1(a). The DC power supply is an important device in the production process of electrolysis. Under the action of DC power supply, $\mathrm{Cu}^{2+}$ in the $\mathrm{CuSO}_{4}$ solution is moved to the negative cathode and forms copper foil on the roller surface. According to the demand of high-grade copper foil, the power supply output must have low voltage but high current.

The HSPS system for electrolytic copper foil is shown in Fig. 1. The HSPS system is composed of 10 HSPS modules in parallel, with the output of a single HSPS module at $6.5 \mathrm{~V} / 5 \mathrm{kA}$. The output capacity of the HSPS system can reach $50 \mathrm{kA}$. The topology of a single HSPS module is shown in Fig. 1(b). The HSPS module is composed of the PWM rectifier and a DC/DC converter. The three-phase two-leg PWM rectifier is adopted to reduce the number of switch devices, and thus reduce the cost and power loss of HSPS. The improved HSPS system has the following features:

1) To enhance the output ability of the HSPS module, two full-wave rectifiers are adopted. The inputs of full-wave rectifiers are in series, but their outputs are in parallel. Thus, the current output of the HSPS module can be enhanced and the capacity utilization of power switches can be improved.

2) By adopting the PWM rectifier, the HSPS can achieve flexible control performance, reduce reactive power, and suppress harmonic pollution.

3) As for the current-sharing issue of the HSPS modules, an improved current-sharing control method is proposed. This method can improve the output characteristic of HSPS modules and achieve the current-sharing operation of the system.

$v_{S a}, v_{S b}, v_{S c}$ are three-phase source voltages, and 
$i_{S a}, i_{S b}, i_{S c}$ are input currents of the PWM rectifier [Fig. 1(b)]. The C-phase source is connected to the midpoint of DC-link capacitors by a filter inductor. Thus, the switch devices only bear half of the DC-link voltage.

According to the circuit shown in Fig. 1(b), the relationship between the voltage and current of the PWM rectifier is as follows:

$$
\left\{\begin{array}{l}
v_{a o}=v_{S a}-v_{S c}-L_{o} \frac{d i_{S a}}{d t}+L_{o} \frac{d i_{S c}}{d t} \\
v_{b o}=v_{S b}-v_{S c}-L_{o} \frac{d i_{S b}}{d t}+L_{o} \frac{d i_{S c}}{d t}
\end{array}\right.
$$

where $L_{o}$ is the input filter inductor. According to the circuit, the following can be obtained:

$$
\left\{\begin{array}{l}
C_{m} * d v_{c 1} / d t=i_{1} \\
C_{m} * d v_{c 2} / d t=i_{2}
\end{array}\right.
$$

where $C_{m}$ is the capacitance of $C_{1}$ and $C_{2} . v_{c 1}$ and $v_{c 2}$ are the voltages of $C_{1}$ and $C_{2}$, and $i_{1}$ and $i_{2}$ are the currents of $C_{1}$ and $C_{2}$ respectively. Meanwhile, the following can be obtained: $i_{S c}=i_{2}-i_{1}$. Assuming $i_{S c}=I_{m} \sin (\omega t+\varphi)$, the following can be obtained:

$$
I_{m} \sin (\omega t+\varphi)=C_{m} * d\left(v_{c 2}-v_{c 1}\right) / d t
$$

Assuming $\Delta u=v_{c 1}-v_{c 2}$. According to Eq. (3), the following can be obtained:

$$
\Delta u=\frac{I_{m}}{C_{m}} \int \sin (\omega t+\varphi) d t=\frac{-I_{m}}{\omega C_{m}} \cos (\omega t+\varphi)+\Delta e=\varepsilon+\Delta e
$$

where $\Delta e$ is the DC component or the DC voltage error of the two capacitors. From Eq. (4), a fundamental AC ripple $\varepsilon$ occurs in the voltage difference, and the $\mathrm{AC}$ component is directly related to $i_{S c}$. Its amplitude is proportional to the magnitude of $i_{S c}$, but inversely proportional to $C_{m}$. In the steady state, the DC-link voltages of the two capacitors can be expressed as follows:

$$
\left\{\begin{array}{l}
v_{c 1}=\left(U_{d}+\varepsilon\right) / 2 \\
v_{c 2}=\left(U_{d}-\varepsilon\right) / 2
\end{array}\right.
$$

where $U_{d}$ is the DC component of the DC-link voltage $u_{d c}$. The voltages of the two DC-link capacitors are easily unbalanced and influenced by disturbances and control errors [24]. Thus, a voltage-balance (VB) controller is always needed to eliminate DC error $\Delta e$. However, traditional VB control directly uses $\Delta u$ as the control input. Hence, the AC component will be introduced into the inner-loop current control. Assuming a proportional controller with a gain $k$ is used for the VB control, the output command signal of the VB controller can be obtained as follows:

$$
i_{\Delta}=k * \Delta u=k \varepsilon+k * \Delta e
$$

In general, the control system will add $i_{\Delta}$ to the current reference of the PWM rectifier. Therefore, an additional instruction $k \varepsilon$ will be added to the current reference. Hence, the normal and stable operation of the PWM rectifier can be seriously affected if $k I_{m} /\left(\omega C_{m}\right)$ is large enough.

\section{CONTROL METHOD OF TWO-LEG PWM RECTIFIER}

According to the model of the PWM rectifier, a composite control method that can effectively combine the merits of feedback control with feedforward control to improve system performance is proposed. The proposed control diagram is shown in Fig. 2. As for the VB control of the PWM rectifier, a ripple filter is adopted to eliminate the $\mathrm{AC}$ ripple and maintain the normal operation of the rectifier.

Assuming the three-phase source voltage and current are as follows:

$$
\begin{aligned}
& \left\{\begin{array}{l}
v_{S a}=U \sin (\omega t) \\
v_{S b}=U \sin (\omega t-2 \pi / 3) \\
v_{S c}=U \sin (\omega t+2 \pi / 3)
\end{array}\right. \\
& \begin{cases}i_{S a}=I \sin (\omega t+\theta) \\
i_{S b}=I \sin (\omega t-2 \pi / 3+\theta) \\
i_{S c}=I \sin (\omega t+2 \pi / 3+\theta)\end{cases}
\end{aligned}
$$

where $U, I$, and $\theta$ are the amplitude of source voltage, source current, and power factor (PF) angle, respectively. In the steady state, the PF angle $\theta$ of the three-phase rectifier is expected to be 0 .

According to the energy balance principle, the system loss of the HSPS is ideally regarded as 0 . Thus, the expected amplitude of the three-phase source currents can be obtained as follows:

$$
I_{S}=2 P_{L} /(3 U)=2 v_{o} * i_{o} /(3 U)
$$

where the load power $P_{L}=v_{o} * i_{o}$, and $v_{o}$ and $i_{o}$ are the load voltage and current respectively. $I_{S}$ can be regarded as the feedforward command signal. Therefore, the PWM rectifier can track the changes of load power in real-time according to $I_{S}$. To maintain the DC-link voltage and compensate for the loss of the power switches, a proportional-integral (PI) controller is adopted for the voltage outer-loop. The output of the voltage controller is $I_{\text {out }}$, and thus the sum $I_{m}=\left(I_{S}+I_{\text {out }}\right)$ can be obtained as shown in Fig. 2. $I_{m}$ is multiplied by the synchronous signals. Hence, the current reference signals for the PWM rectifier can be obtained as follows:

$$
\left\{\begin{array}{l}
i_{a r}=I_{m} * \sin (\omega t) \\
i_{b r}=I_{m} * \sin (\omega t-2 \pi / 3)
\end{array}\right.
$$

Assuming the output currents and voltages of PWM rectifier are balanced in the steady state, the sum of three-phase source currents is zero because no neutral line exists. Thus, the C-phase expected current can be obtained as 


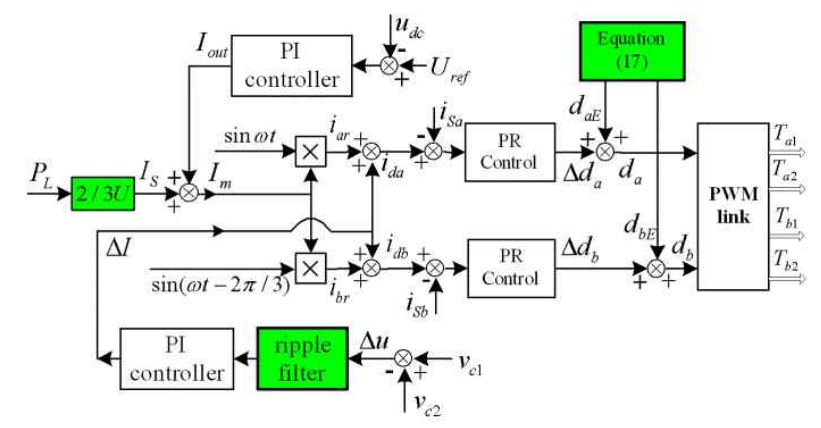

Fig. 2. Composite control diagram of the two-leg PWM rectifier.

follows:

$$
i_{c r}=-\left(i_{a r}+i_{b r}\right)=I_{m} * \sin (\omega t+2 \pi / 3)
$$

As for the VB control of the PWM rectifier, a PI controller is adopted. According to the discussion above, a fundamental AC ripple $\varepsilon$ occurs in the voltage difference $\left(v_{c 1}-v_{c 2}\right)$, which is caused by $i_{S c}$. According to Eq. (11), the fundamental AC ripple $\varepsilon$ can be obtained as follows:

$$
\varepsilon=-I_{m} \cos (\omega t+2 \pi / 3) /\left(\omega C_{m}\right)
$$

$\varepsilon$ can be subtracted from $\Delta u$ to obtain the DC error $\Delta e$ and eliminate the AC ripple in $\Delta u$ (Fig. 2). Thus, the pure DC component $\Delta e$ can be obtained and processed by a PI controller. A command signal $\Delta I$ for voltage balance can then be obtained. The voltage-balance command $\Delta I$ can change the input current of the PWM rectifier to intentionally strengthen or weaken the charging of some capacitor. Finally, the dynamic balance of two capacitors can be achieved.

The total reference signals of current inner-loop can be obtained as follows:

$$
\left\{\begin{array}{l}
i_{d a}=i_{a r}+\Delta I \\
i_{d b}=i_{b r}+\Delta I
\end{array}\right.
$$

With the traditional PI controller, tracking ability is limited for the AC signal, and error will occur in the steady state. Thus, a proportional resonant (PR) controller that can effectively improve the control error was proposed [25]. The $s$-domain of the PR controller is expressed as follows:

$$
G(s)=k_{g}+2 k_{i} s /\left(s^{2}+\omega^{2}\right)
$$

where $k_{g}$ is the proportional coefficient, and $k_{i}$ is the integral coefficient. The PR feedback control can output the dynamic adjustment signals ( $\Delta d_{a}, \Delta d_{b}$ ) (Fig. 2).

According to Eq. (1) and the average switch principle of the bipolar PWM, the output voltages of $v_{a o}$ and $v_{b o}$ can be derived as follows:

$$
\left[\begin{array}{l}
v_{a o} \\
v_{b o}
\end{array}\right]=\left[\begin{array}{l}
v_{S a}-v_{S c}-L_{o} \frac{d}{d t}\left(2 i_{S a}+i_{S b}\right) \\
v_{S b}-v_{S c}-L_{o} \frac{d}{d t}\left(i_{S a}+2 i_{S b}\right)
\end{array}\right]=\left[\begin{array}{l}
\left(2 d_{a}-1\right) \frac{u_{d c}}{2} \\
\left(2 d_{b}-1\right) \frac{u_{d c}}{2}
\end{array}\right]
$$

where $d_{a}$ and $d_{b}$ are the duty cycles of the two-leg PWM

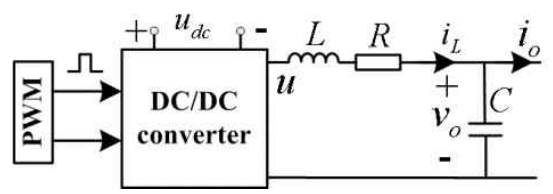

Fig. 3. Equivalent model of single DC/DC converter.

rectifier, and $d_{a}$ and $d_{b} \in[0,1]$ occur. Then, the duty cycles can be expressed as follows:

$$
\left\{\begin{array}{l}
d_{a}=\frac{1}{u_{d c}} v_{S a c}-\frac{2 L_{o}}{u_{d c}} \frac{d i_{S a}}{d t}-\frac{L_{o}}{u_{d c}} \frac{d i_{S b}}{d t}+\frac{1}{2} \\
d_{b}=\frac{1}{u_{d c}} v_{S b c}-\frac{L_{o}}{u_{d c}} \frac{d i_{S a}}{d t}-\frac{2 L_{o}}{u_{d c}} \frac{d i_{S b}}{d t}+\frac{1}{2}
\end{array}\right.
$$

where $v_{S b c}$ and $v_{S b c}$ are the source line voltages. With the expected currents shown as Eq. (10), according to Eq. (16), the expected duty cycles $d_{a E}$ and $d_{b E}$ of the two-leg PWM rectifier can be calculated as Eq. (17).

Deriving Eq. (17) is based on the switch model of the PWM rectifier; the model Eq. (17) can directly control the rectifier output the corresponding voltage to obtain the expected current. Hence, the expected duty cycles $d_{a E}$ and $d_{b E}$ can be used as the feedforward control signals to realize a rapid response for current control.

$$
\left\{\begin{array}{l}
d_{a E}=\frac{v_{S a c}}{u_{d c}}-\frac{2 \omega I_{m} L_{o}}{u_{d c}} \cos \omega t-\frac{\omega I_{m} L_{o}}{u_{d c}} \cos \left(\omega t-\frac{2 \pi}{3}\right)+\frac{1}{2} \\
d_{b E}=\frac{v_{S b c}}{u_{d c}}-\frac{\omega I_{m} L_{o}}{u_{d c}} \cos \omega t-\frac{2 \omega I_{m} L_{o}}{u_{d c}} \cos \left(\omega t-\frac{2 \pi}{3}\right)+\frac{1}{2}
\end{array}\right.
$$

Finally, the total duty cycles $d_{a}$ and $d_{b}$ of the PWM rectifier can be calculated by superimposing the outputs of feedback control with the feedforward control. Thus, the control system of the PWM rectifier effectively combines the close-loop tracking performance of the feedback control with the rapid response of the feedforward control, which can effectively improve the operation performance of the system.

\section{CONTROl Method of DC/DC CONVERTER}

The equivalent model of single DC/DC converter is shown in Fig. 3. $L$ and $C$ are the parameters of output filter, and $R$ is the equivalent series resistance corresponding to the loss.

Given that copper-foil electrolysis is a dynamic engineering process, the solution in the electrolytic tank is prone to fluctuations. Hence, the equivalent resistance of load easily varies, which will affect the stable operation of the power supply. The load current is considered a disturbance because the load varies. The control system of the single DC/DC converter in $s$ domain is established (Fig. 4).

In Fig. $4, F_{i}$ is the load current feedforward function, and $H_{i}$ and $H_{v}$ are the feedback links that can be considered a first filter $1 /\left(\tau_{C} s+1\right)$, where $\tau_{C}$ is the time constant of the 


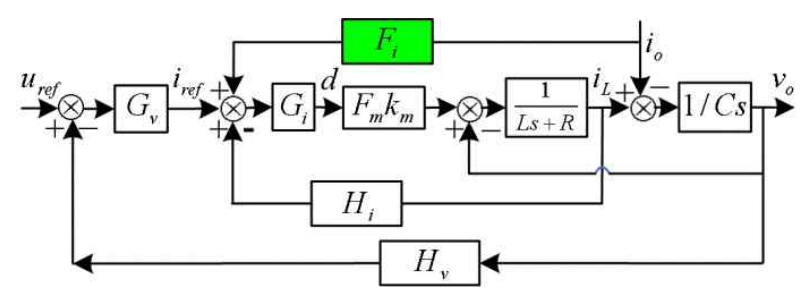

Fig. 4. Control diagram with load current feedforward.

filter. $G_{i}$ and $G_{v}$ are the current and voltage controllers respectively. Here, $G_{i}$ is a proportional controller with a gain $k_{c}$, while, $G_{v}$ is a PI controller with the expression $k_{p}\left[1+1 /\left(T_{i} s\right)\right]$, where $k_{p}$ and $T_{i}$ are the gain and integral coefficient respectively. $k_{m}$ is the gain of the DC/DC converter, and it is $U_{d} /(2 n)$, where $n$ is the turn ratio of the transformer. $F_{m}$ is $1 / V_{m}$, and $V_{m}$ is the amplitude of the carrier wave.

The output voltage of the HSPS can be expressed as

$$
v_{o}=G_{u} u_{r e f}-Z_{o} i_{o}
$$

According to Fig. 4, the following can be obtained:

$$
\left\{\begin{array}{l}
G_{u}=v_{o} / u_{r e f}=G_{v} G_{i} k_{m} F_{m} / A(s) \\
Z_{o}=-v_{o} / i_{o}=\left[-F_{i} G_{i} k_{m} F_{m}+H_{i} G_{i} k_{m} F_{m}+L s+R\right] / A(s)
\end{array}\right.
$$

where $A(s)=H_{v} G_{v} G_{i} k_{m} F_{m}+H_{i} G_{i} k_{m} F_{m} C s+(L s+R) C s+1$.

To suppress the output voltage fluctuations caused by load changes and increase the voltage output ability of power supply, a load current full feedforward method is adopted as shown in Eqs. (18) and (19). This method is used to control the equivalent output impedance $Z_{\mathrm{o}}$ approach to 0 , which can suppress the adverse effect of load changes. Therefore, when $Z_{\mathrm{o}}$ is set as 0 , the following can be derived:

$$
F_{i}=H_{i}+\frac{(L s+R)}{G_{i} k_{m} F_{m}}=\frac{L \tau_{C} s^{2}+\left(L+R \tau_{C}\right) s+R+G_{i} k_{m} F_{m}}{G_{i} k_{m} F_{m}\left(\tau_{C} s+1\right)}
$$

Given that $\tau_{C}$ is small and $R \ll G_{i} k_{m} F_{m}$, to facilitate the implementation of the controller, Eq. (20) can be simplified as follows:

$$
F_{i}=\frac{L_{D} s+1}{\left(\tau_{C} s+1\right)}, \quad L_{D}=L /\left(k_{c} k_{m} F_{m}\right)
$$

According to the control and circuit parameters of the DC/DC converter (Table I), the Bode diagram of $Z_{\mathrm{o}}$ is shown in Fig. 5. When $F i=0$, the load current feedforward method is not used. After using the load current feedforward method $(F i=1)$ [17], the gain of equivalent output impedance $Z_{\mathrm{o}}$ decreases in the low-frequency band. Then, using the proposed full feedforward function, the gain of $Z_{\mathrm{o}}$ is attenuated greatly in the low-frequency range. Thus, this method can effectively enhance the ability of load anti-disturbance and increase the voltage output capacity of the power supply.

\begin{tabular}{|c|c|}
\hline Parameter name & Value \\
\hline \multicolumn{2}{|c|}{ System parameters } \\
\hline Source voltage & $220 \mathrm{~V}, 50 \mathrm{~Hz}$ \\
\hline AC filter inductor & $L_{\mathrm{o}}=1 \mathrm{mH}$ \\
\hline DC-link capacitors & $\mathrm{C}_{1}=\mathrm{C}_{2}=10000 \mathrm{uF}$ \\
\hline $\begin{array}{l}\text { High-frequency } \\
\text { transformer }\end{array}$ & $n=40: 1$ \\
\hline Output inductor & $L=0.08 \mathrm{mH}$ \\
\hline Output capacitor & $C=3000 \mathrm{uF}$ \\
\hline \multicolumn{2}{|c|}{ Control parameters of the PWM rectifier } \\
\hline Voltage controller & $2(1+0.01 \mathrm{~s}) / 0.01 \mathrm{~s}$ \\
\hline VB controller & $2(1+0.01 \mathrm{~s}) / 0.01 \mathrm{~s}$ \\
\hline Current controller & $10+150 \mathrm{~s} /(\mathrm{s} * \mathrm{~s}+98696)$ \\
\hline \multicolumn{2}{|c|}{ Control parameters of the DC/DC converter } \\
\hline Current controller & 0.2 \\
\hline Voltage controller & $10(1+0.05 \mathrm{~s}) / 0.05 \mathrm{~s}$ \\
\hline
\end{tabular}

The power supply system for electrolytic copper foil is composed of multi-parallel power modules. Given the
TABLE I

PARAMETERS OF THE HSPS

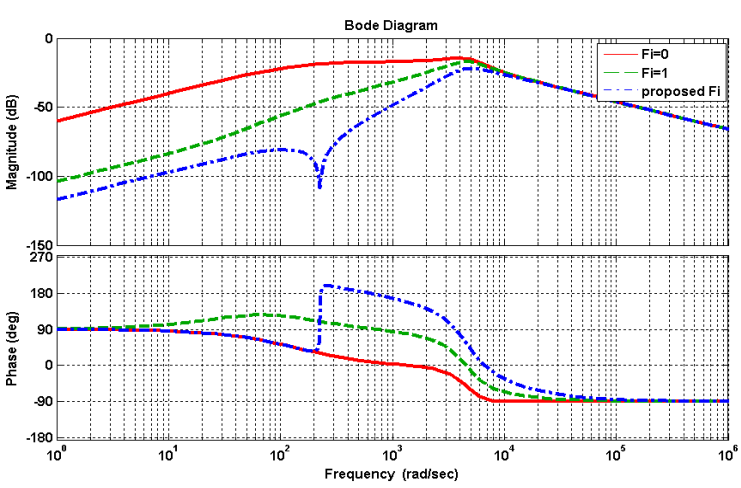

Fig. 5. Bode diagram of equivalent inner impedance $Z_{\mathrm{o}}$

differences in system parameters of each power module, current-sharing error will be produced. According to [18], the output voltage $u$ of converter can be expressed as follows:

$$
u=\frac{U_{d}}{2 n} * D-\frac{U_{d}}{2 n} *\left(2 D_{d}+D_{o f f}\right)-U_{S e}
$$

where $D$ is the normal duty ratio of the converter, $U_{S e}$ is the voltage drop of switches (diodes) and, $D_{d}$ and $D_{\text {off }}$ are the loss of duty ratio caused by the dead time and soft-switching respectively, which are important factors that cause output voltage errors. In Fig. $6, u_{e}$ is the offset voltage caused by $U_{S e}, D_{d}$, and $D_{o f f}$. To achieve the current-sharing control, a control method based on the virtual resistor is proposed. $R_{v}$ is the virtual resistor, which is the control gain of current-sharing loop. $i_{a v}$ is the average output current of multi-parallel power modules. $i_{h}$ is the current-sharing error. According to Fig. 6, the following can be obtained:

$$
\left\{\begin{array}{l}
Z_{h}=v_{o} / i_{h}=R_{v} G_{v} G_{i} k_{m} F_{m} / A(s) \\
G_{e}=-v_{o} / u_{e}=1 / A(s)
\end{array}\right.
$$




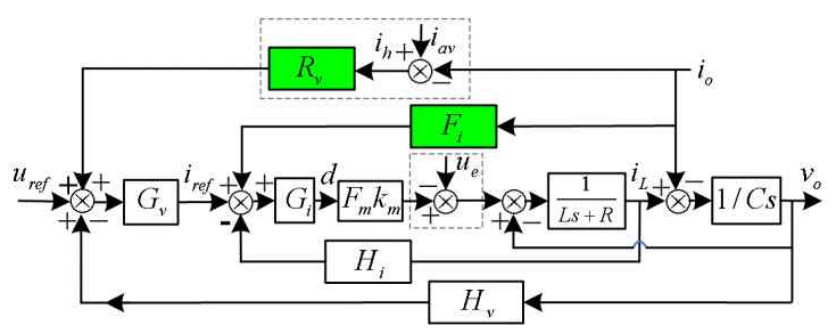

Fig. 6. Current-sharing control method of the DC/DC converter.

where $Z_{\mathrm{h}}$ can be defined as the current-sharing impedance. The current-sharing control will retain the stability of the closed-loop control system and the value of the equivalent output impedance $Z_{\mathrm{o}}$. Thus, this implementation method of virtual impedance is through the feedback of current-sharing error, which offers better performance than the method through the feedback of inductive current. Assuming $G_{u}, Z_{o}$, $G_{e}$, and $Z_{h}$ are similar for m-parallel power modules, the output voltage expression of $\mathrm{j}$-th power module can be obtained as follows:

$$
v_{o}=G_{u} u_{r e f j}-Z_{o} i_{o j}-G_{e} u_{e j}+Z_{h} i_{h j}
$$

Then, according to Eq. (24), the following can be obtained:

$$
\text { where }\left\{\begin{array}{l}
v_{o}=G_{u} u_{\text {refav }}-Z_{o} i_{a v}-G_{e} u_{e a v} \\
i_{a v}=\frac{1}{m} \sum u_{r e f j} ; u_{e a v}=\frac{1}{m} \sum u_{e j} ; 0=\frac{1}{m} \sum i_{h j}
\end{array}\right.
$$

$m$ is the number of power modules. If Eq. (24) is subtracted from Eq. (25), the following can be obtained:

$$
\left(Z_{o}+Z_{h}\right) i_{h j}=G_{u}\left(u_{r e f a v}-u_{r e f j}\right)-G_{e}\left(u_{e a v}-u_{e j}\right)
$$

Then, the following can be derived:

$$
i_{h j}=\frac{G_{u}}{\left(Z_{o}+Z_{h}\right)}\left(u_{r e f a v}-u_{r e f j}\right)-\frac{G_{e}}{\left(Z_{o}+Z_{h}\right)}\left(u_{e a v}-u_{e j}\right)
$$

In practice, the voltage references given for power modules are generally similar, that is, the value of the first term in Eq. (27) is approximately 0 . However, the offset voltage $u_{\mathrm{ej}}$ of each power module varies with different parameters of switches and dead time. This phenomenon is the major factor causing current-sharing error. In the case of high-power operation in particular, given that the system impedance is small, a small bias voltage will produce a large current-sharing error. The smaller the $Z_{0}$, the poorer the inhibitory effect on $i_{\mathrm{h}}$, as shown in Eq. (27). Therefore, in the case that $Z_{\mathrm{o}}$ is determined, selecting the virtual resistance $R_{\mathrm{v}}$ to decay the gain of $G_{\mathrm{e}} /\left(Z_{\mathrm{o}}+Z_{\mathrm{h}}\right)$ can be reasonable. Thus, the current-sharing error caused by the offset voltage error can be eliminated through this method. According to Eqs. (23) and (19), the following can be obtained:

$$
\frac{G_{e}}{\left(Z_{o}+Z_{h}\right)}=\frac{1}{H_{i} G_{i} k_{m} F_{m}+L s+R-F_{i} G_{i} k_{m} F_{m}+R_{v} G_{v} G_{i} k_{m} F_{m}}
$$

According to Eq. (28) and the parameters of DC/DC converter (Table I), the Bode diagram of $G_{\mathrm{e}} /\left(Z_{\mathrm{o}}+Z_{\mathrm{h}}\right)$ is shown

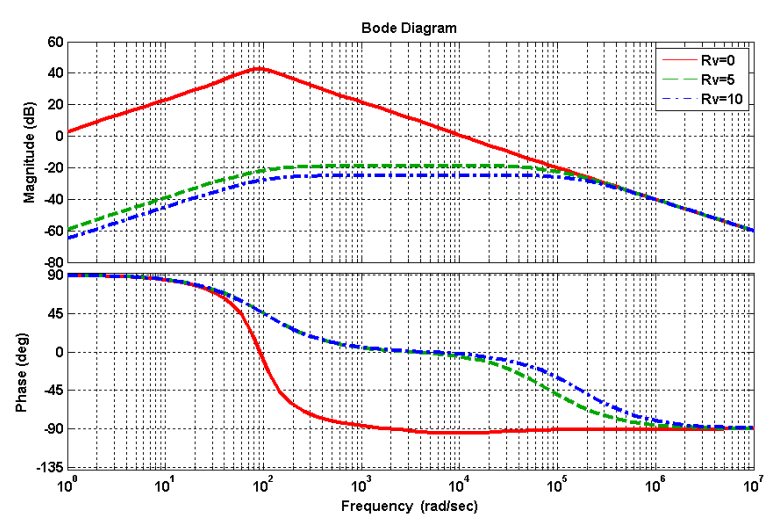

Fig. 7. Bode diagram of $\mathrm{G}_{\mathrm{e}} /\left(\mathrm{Z}_{\mathrm{o}}+\mathrm{Z}_{\mathrm{h}}\right)$.

in Fig. 7. When the virtual resistor is set as 0 , the gain of $G_{\mathrm{e}} /\left(Z_{\mathrm{o}}+Z_{\mathrm{h}}\right)$ is large in the low-frequency band. Hence, a small bias voltage will generate a significant current-sharing error. When the virtual resistance is 5 , the gain of $G_{\mathrm{e}} /\left(Z_{\mathrm{o}}+Z_{\mathrm{h}}\right)$ is attenuated substantially. Thus, the current-sharing error in low-frequency range will be greatly reduced. With increasing virtual resistance, the current-sharing impedance $Z_{\mathrm{h}}$ can be effectively increased, which can attenuate the gain of $G_{\mathrm{e}} /\left(Z_{\mathrm{o}}+Z_{\mathrm{h}}\right)$. Here, the value of $R_{\mathrm{v}}$ is selected as 10 . In summary, the control system has an inhibitory effect on both DC and harmonic bias voltage, and the virtual resistance $R_{\mathrm{v}}$ has a significant suppression effect on the low-frequency bias voltage.

\section{SimUlations}

To verify that the proposed system is feasible, the simulation model is established by using PSIM6 software (Fig. 1). The design of the HSPS system is as follows: AC line voltage is $220 \mathrm{~V} / 50 \mathrm{~Hz}$, and output of the HSPS module is DC $5 \mathrm{kA} / 6.5 \mathrm{~V}$ for copper-foil electrolysis. The DC-link voltage of the PWM rectifier is set to $700 \mathrm{~V}$, and the turn ratio of the transformer is 40:1. The switching frequencies $f_{\mathrm{s}}$ of the PWM rectifier and full-bridge inverter are 10 and $20 \mathrm{kHz}$ respectively. The amplitude of carrier wave $V_{\mathrm{m}}=10$. A resistor $\left(R_{\mathrm{d}}=6.5 / 5 \mathrm{k}\right)$ is used to simulate the load. The other circuit parameters of the HSPS are shown in Table I.

The load is switched into the system at $0.4 \mathrm{~s}$, and the output voltage reference is linearly given from $0 \mathrm{~V}$ at $0.4 \mathrm{~s}$ to $6.5 \mathrm{~V}$ at $0.6 \mathrm{~s}$. Fig. 8(a) shows the output voltage and current waveforms of the power module. The output voltage "Vo" of the DC/DC converter almost linearly increases with the reference "uref." Thus, the output voltage can fast track to the reference without vibration. In the steady state, the output voltage and current of DC/DC converter are stable at the expected value [Fig. 8(a)]. Fig. 8(b) shows the dynamic current and voltage waveforms of the PWM rectifier. With increasing output voltage and current, the load power increases. Hence, the input currents of the PWM rectifier also rapidly increase with increasing output power. Meanwhile, 

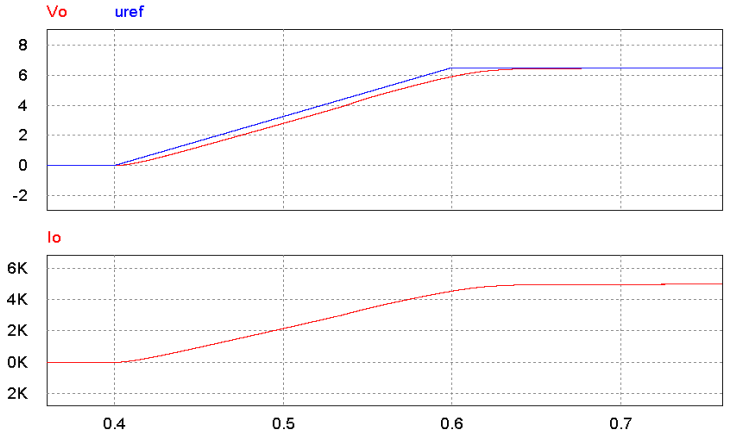

(a)
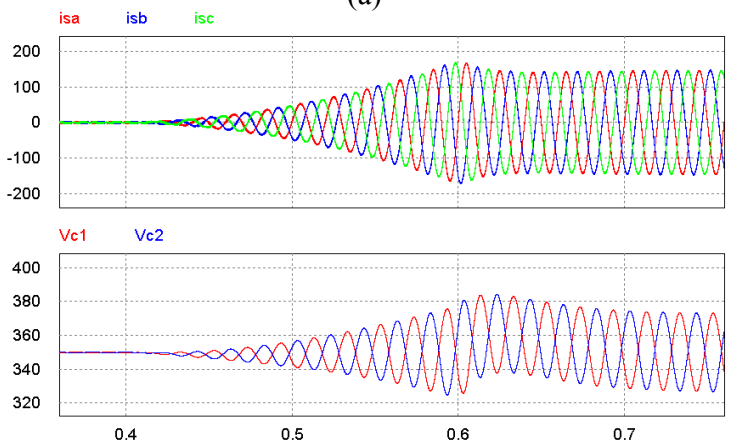

(b)

Fig. 8. Dynamic waveforms. (a) Output waveforms of the DC/DC converter. (b) Waveforms of the PWM rectifier.

the $\mathrm{AC}$ voltage ripples of the DC-link capacitors also increase with increasing C-phase input current of the PWM rectifier [Fig. 8(b)].

To confirm the proposed ripple filter for the VB control, a comparison simulation is conducted. Fig. 9(a) shows the detected voltage difference and voltage ripple "um" calculated by using Eq. (12) in the steady state. The voltage ripple "um" is nearly similar to the detected voltage difference, and their amplitude is approximately $43 \mathrm{~V}$.

A fundamental ripple caused by the C-phase current of the PWM rectifier occurs in the voltage difference. If the voltage ripple is not eliminated, then this ripple will be processed by the VB controller and added to the current instructions of the PWM rectifier. With the current inner-loop control, the voltage ripple will generate a corresponding current in the input currents of the PWM rectifier. Thus, the PWM rectifier will be out of normal operation [Fig. 9(b)]. If the control system adopts the ripple filter proposed in this paper, it can eliminate the fundamental ripple in the voltage difference without adding a low-pass filter, and the VB controller can then eliminate the DC error of voltage difference. In the steady state, the PWM rectifier can operate normally, and the input currents are balanced at 93.5 A [Fig. 9(c)]. The PF of the PWM rectifier is 0.99 , and total harmonic distortion (THD) of the current is $3.2 \%$. Thus, the proposed HSPS can effectively reduce harmonic currents and improve power quality of the system.

A simulation is conducted (Fig. 10) to confirm the performance of the proposed control method with
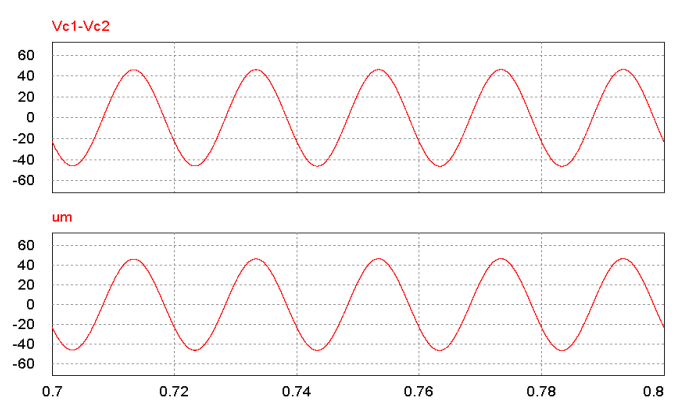

(a)
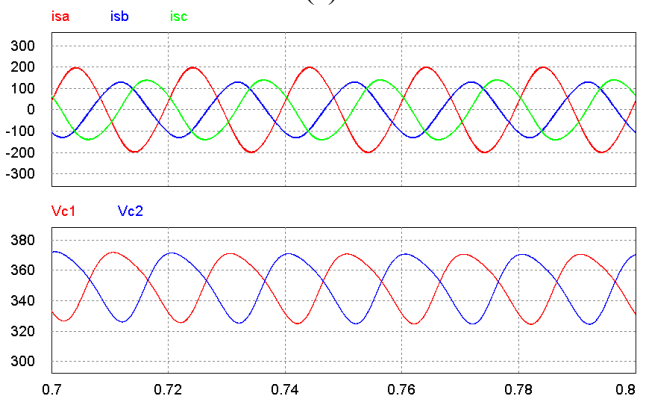

(b)
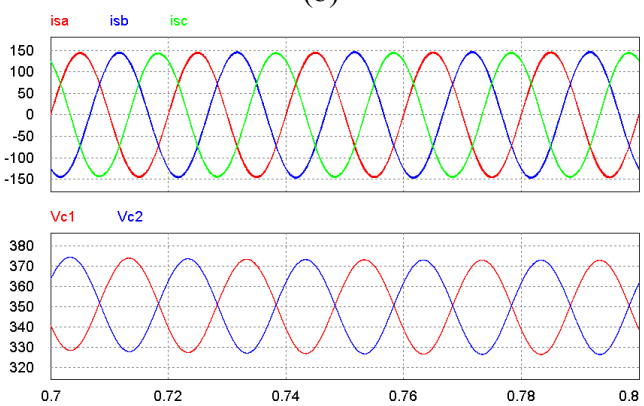

(c)

Fig. 9. Comparison of waveforms for the ripple filter. (a) Voltage difference of DC-link capacitors. (b) Waveforms without ripple filter. (c) Waveforms with ripple filter.

feedforward control. A disturbance of $0.05 \Omega$, which is in parallel with the load, is switched into the system at $0.7 \mathrm{~s}$ and switched off at $0.71 \mathrm{~s}$.

As the disturbance is switched into the system, the output voltage and current of the HSPS suddenly change. However, with the proposed control method, the output current can rapidly attenuate to the steady-state value within $0.02 \mathrm{~s}$ [Fig. 10(a)]. As the load changes, the output power also varies, which can result in fluctuations of the DC-link voltage and input current of the PWM rectifier. Given that the PWM rectifier adopts the proposed method, two kinds of feedforward control signals exist. One is the feedforward of load power, by which it can immediately calculate the current instructions according to Eqs. (9) and (10) for current controller. The other is the feedforward of duty cycles calculated from Eq. (17). Thus, the control system can rapidly obtain the expected duty cycles of the PWM rectifier, which can cause it to output the expected $\mathrm{AC}$ voltages to rapidly generate the corresponding currents [Fig. 10(b)].

To present the results of other conditions, a disturbance of 


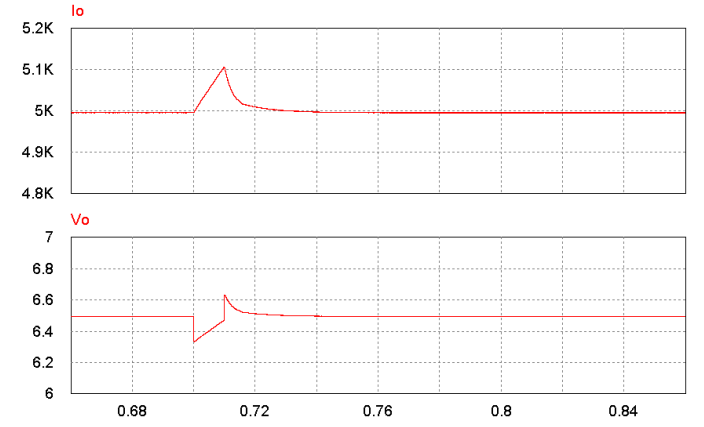

(a)

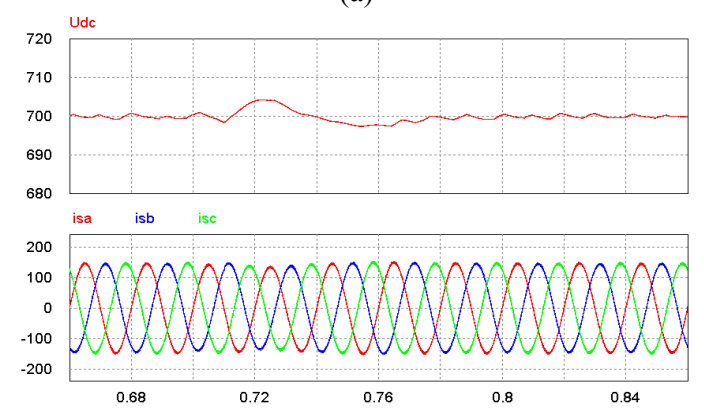

(b)

Fig. 10. Dynamic waveforms with a disturbance of $0.05 \Omega$ : (a) waveforms of the DC/DC converter, (b) waveforms of the PWM rectifier.

$0.025 \Omega$ is switched into the system at $0.8 \mathrm{~s}$ and switched off at 0.9 s. The results are shown in Fig. 11.

When the disturbance occurs, the output voltage falls down or ascends suddenly [Fig. 11(a)]. With the proposed feedforward control method of DC/DC converter, the output voltage increases or descends rapidly to track the reference without vibrations. In general, DC-link capacitors can be considered a large inertia link. As the output load power changes severely, the DC-link voltage will fluctuate. With the proposed control method, the PWM rectifier can rapidly regulate the output voltage and current to charge or discharge the capacitors and maintain a stable voltage [Fig. 11(b)].

To verify the current-sharing control method of the DC/DC converter based on virtual resistance, dead time is used to simulate the parameter difference of the HSPS modules. The dead time of one HSPS module is set as 1 us, whereas the other is set as 3 us. The other circuit and control parameters of HSPS module are similar. The open-loop output voltages of two power modules and their spectrum when the duty ratio is set to 0.8 are shown in Fig. 12 .

Dead time can cause the loss of output voltage (Fig. 12). The larger the dead time, the greater the output voltage loss. In the spectrum of output voltages, a $0.2 \mathrm{~V} \mathrm{DC}$ offset voltage occurs between them which will generate a non-negligible current-sharing error.

To verify the performance of the proposed current-sharing control based on virtual resistance, a simulation comparison is conducted (Fig. 13). At the first time, the dead time of two-parallel power modules is set as $1 \mathrm{us,} \mathrm{and} \mathrm{they} \mathrm{share} \mathrm{with}$
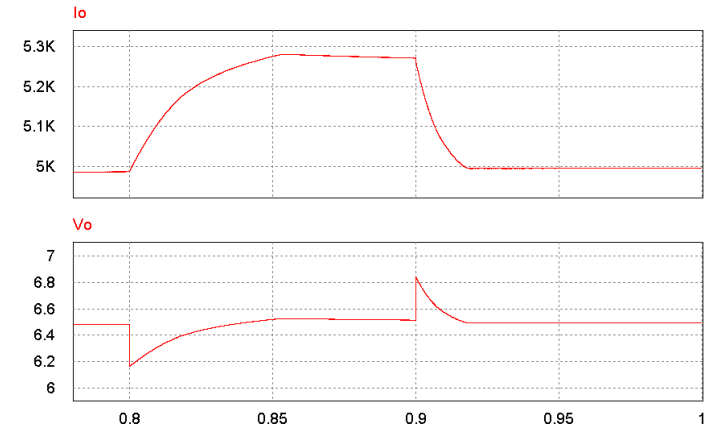

(a)

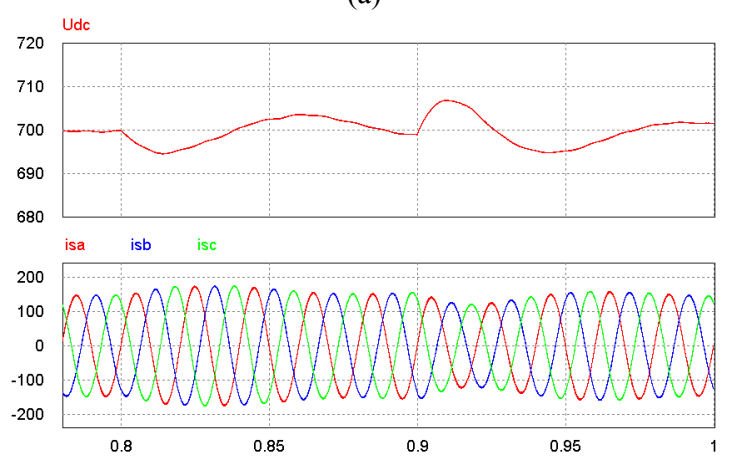

(b)

Fig. 11. Dynamic waveforms with a disturbance of $0.025 \Omega$ : (a) waveforms of the DC/DC converter, (b) waveforms of the PWM rectifier.

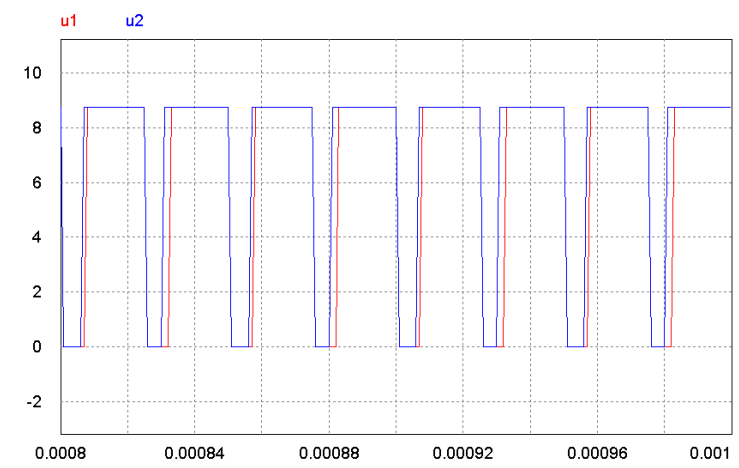

(a)

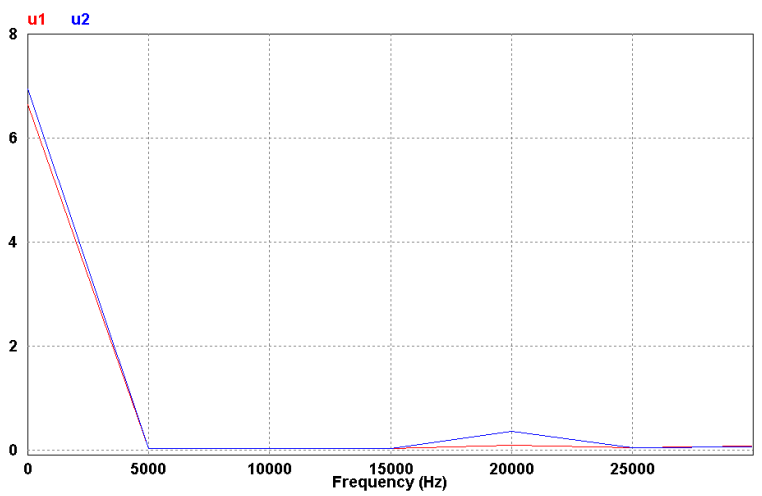

(b)

Fig. 12. Open-loop output voltages with different dead time: (a) output voltages, (b) spectrum of the output voltages.

the load. The dead time of one HSPS module is changed to 3 us at $0.8 \mathrm{~s}$ to compare the dynamic current-sharing control 


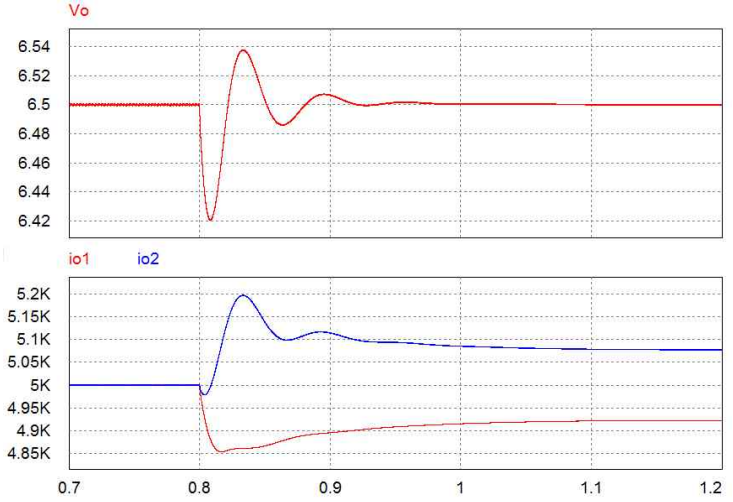

(a)

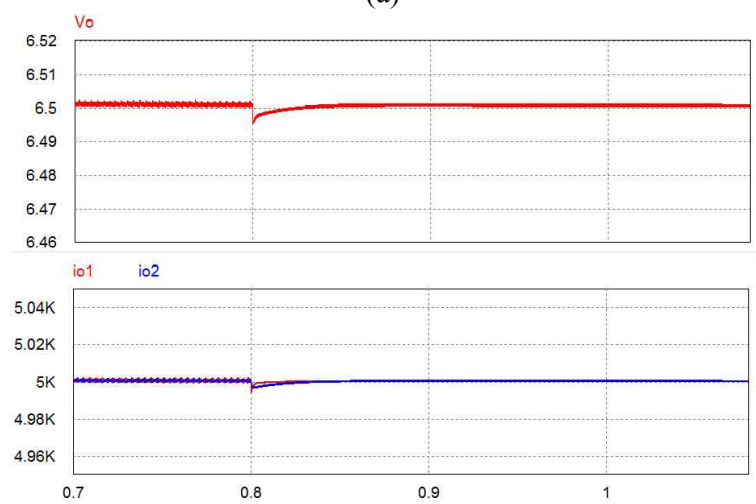

(b)

Fig. 13. Output waveforms of the two HSPSs with two different control methods. (a) Waveforms with traditional dual-loop control method. (b) Waveforms with the proposed control method.

\section{performance.}

Fig. 13(a) shows the dynamic output voltage and current waveforms of the two HSPSs with traditional dual-loop control method with current-sharing control loop. Before the dead time disturbance, the output voltage is stable at $6.5 \mathrm{~V}$ and the output currents of the two HSPSs are stable at the expected value of $5 \mathrm{kA}$. When the dead time is the same, almost no current-sharing error occurs (io1 $\approx$ io 2 ). As the dead time disturbance occurs at $0.8 \mathrm{~s}$, a dynamic adjustment process with a few vibrations will occur before the system stabilizes. In the steady state, a current-sharing error of $77 \mathrm{~A}$ occurs for the output currents of the two HSPSs.

The waveforms when using the proposed control method with the dead-time disturbances at $0.8 \mathrm{~s}$ are shown in Fig. 13(b). Given that the current-sharing control loop can rapidly regulate the output voltages of the two HSPSs, the output vibrations can be effectively suppressed and rapidly go into the steady state. According to the analysis above, with the closed-loop control of virtual resistance, the gain of current-sharing impedance $Z_{\mathrm{h}}$ can increase to effectively attenuate the gain $G_{\mathrm{e}} /\left(Z_{\mathrm{o}}+Z_{\mathrm{h}}\right)$. This method can greatly reduce the current-sharing error caused by the offset voltage. In the steady state, the current-sharing error of the HSPS modules is almost 0 [Fig. 13(b)]. Thus, the proposed control method can

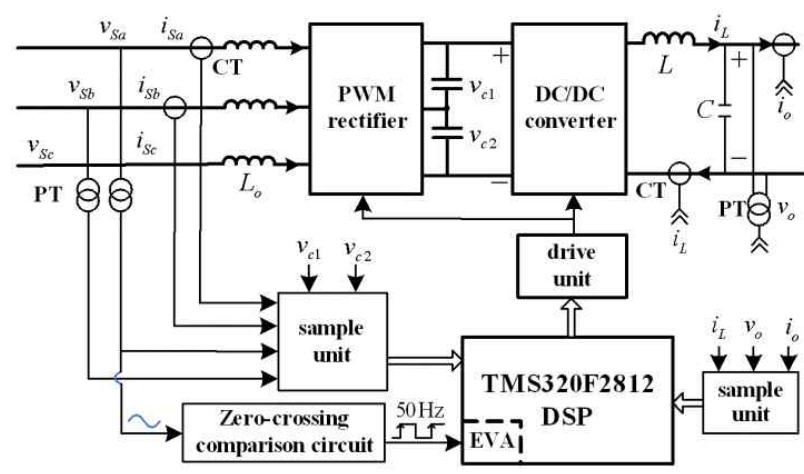

(a)

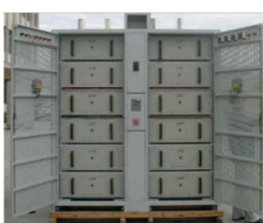

(b)

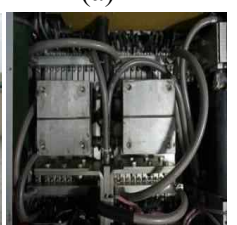

(c)

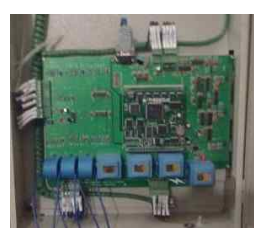

(d)
Fig. 14. Implementation figures of the HSPS system. (a) Physical implementation diagram. (b) Physical photo of system. (c) High-frequency transformer. (d) DSP control board.

effectively improve the current-sharing performance of the HSPS system.

\section{EXPERIMENT AND INDUSTRIAL APPLICATION}

To verify the feasibility of the structure and the proposed control method, an industrial prototype of $220 \mathrm{~V}$ HSPS system is developed. For high-precision electronic copper project, the output of DC power supply must be $6.5 \mathrm{~V} / 50 \mathrm{kA}$. Therefore, an HSPS system composed of 10 parallel 6.5 V/5 kA HSPS modules (Fig. 1) is developed. The design and circuit parameters of the HSPS module are listed above.

Each HSPS module adopts a TMS320F2812 as the digital signal processor (DSP), which is used to detect signals, execute algorithm, and generate the PWM drive signals for the IGBTs of the PWM rectifier and full-bridge inverter. The physical implementation diagram of the HSPS is shown in Fig. 14(a). The A-phase voltage is processed by the zero-crossing comparison circuit, and then the rising edge of output square wave is used to trigger an interruption by event manager A (EVA) of DSP. Thus, the current phase-angle of the PWM rectifier can be initialized in every grid period. The physical photos of the device are shown in Figs. 14(b) to 14(d). The field experimental waveforms with the proposed control method are shown in Figs. 15 to 16.

The input currents of the HSPS module are stable at $94.3 \mathrm{~A}$ [Fig. 15(a)]. The voltages of the DC-link capacitors are stable at $350 \mathrm{~V}$ [Fig. 15(b)]. The current waveforms are smooth with a THD of $4.3 \%$, and the PF of the PWM rectifier is about 0.98 . The power quality of the system is effectively improved compared with the uncontrolled rectifier. In the steady state, the load voltage is stable at $6.5 \mathrm{~V}$ [Fig. 15(c)], 


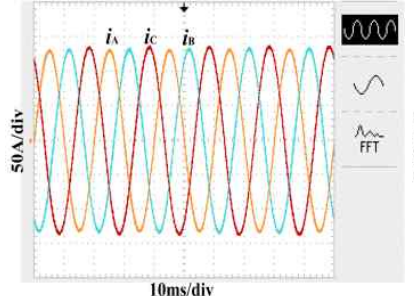

(a)

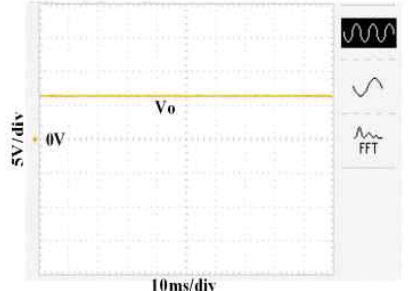

(c)

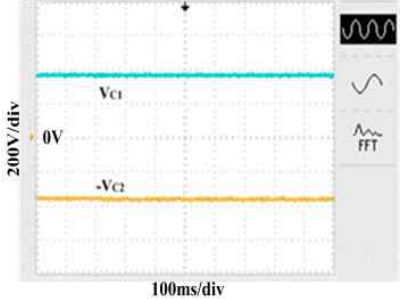

(b)

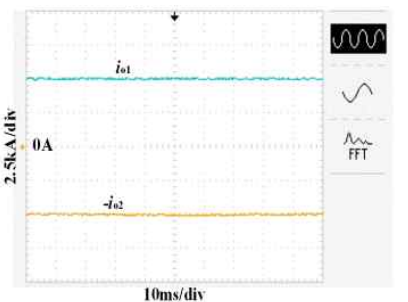

(d)
Fig. 15. Experimental waveforms. (a) Three-phase input currents. (b) DC-link voltages. (c) Output DC-voltage of system. (d) Output DC-currents.

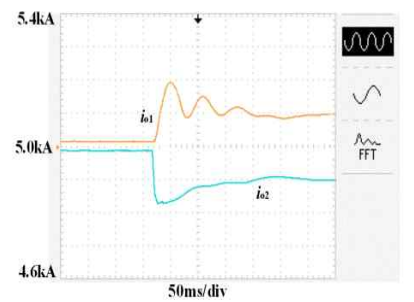

(a)

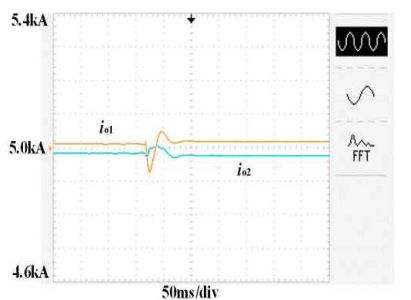

(b)
Fig. 16. Experimental waveforms for current-sharing. (a) Output currents with traditional dual-loop control. (b) Output currents with the proposed current-sharing control.

TABLE II

ERRORS OF OUTPUT CURRENT OF 10 HSPS MODULES

\begin{tabular}{ccccccccccc}
\hline \hline $\begin{array}{c}\text { Error } \\
\text { (unit:A) }\end{array}$ & 15 & 5 & -13 & 8 & -10 & 18 & -3 & 16 & -24 & -7 \\
\hline $\begin{array}{c}\text { module } \\
\text { number }\end{array}$ & 1 & 2 & 3 & 4 & 5 & 6 & 7 & 8 & 9 & 10 \\
\hline
\end{tabular}

and the output currents of any two modules in the HSPS system are stable at about $5 \mathrm{kA}$ [Fig. 15(d)].

An experimental comparison is conducted to further verify the proposed current-sharing control method. The experimental condition is similar to that in Fig. 13.

As the dead time of one HSPS module changes, a current-sharing error occurs using the traditional dual-loop control method [Fig. 16(a)]. When using the proposed current-sharing control method based on virtual resistance, the current-sharing error is greatly reduced [Fig. 16(b)]. These experimental results are similar to those in Fig. 13. Given the dispersion of circuit parameters $(L, C$, and $R)$ of the prototype, the current-sharing error is not 0 in the steady state.

The errors of output currents (expected value: $5 \mathrm{kA}$ ) of the 10 HSPS modules with the proposed control method are shown in Table II. The maximum error is $-24 \mathrm{~A}$ and less than $0.5 \%$. Thus, the output of the HSPS modules is almost current sharing, and the system can operate stably by using the proposed control method. This proposed HSPS system can achieve high-quality rectification and high-current output to meet the requirements of electrolysis.

The proposed HSPS technology and its device have been successfully installed on the industrial production line of an $18 \mu \mathrm{m}$ electrolytic copper foil in Jiangxi Copper Group, Southeast China. Compared with the traditional thyristor power supply, the efficiency is increased from $75 \%$ to $90 \%$ and the output current error is reduced from $2 \%$ to $0.5 \%$. Given that the control performance of HSPS system is effectively enhanced, the quality of an $18 \mu \mathrm{m}$ copper foil is greatly improved and the rate of the finished products is increased by $10 \%$.

\section{CONCLUSION}

To meet the requirements of copper-foil electrolysis, a 6.5 V/50 kA HSPS system based on PWM rectifier is investigated. As for the VB control of the PWM rectifier, a ripple filter method is proposed to eliminate the AC ripple. According to the model of the PWM rectifier, a composite control method is proposed to improve the control performance of the system. To meet the process requirements of electrolysis, a control method with load current full feedforward is proposed for DC/DC converters, which can decay the equivalent output impedance $Z_{\mathrm{o}}$ to enhance the anti-disturbance ability. Aiming at current-sharing of a multi-parallel system, a current-sharing control method based on virtual resistance is proposed. This method can adjust the current-sharing impedance $Z_{\mathrm{h}}$ by virtual resistance to damp the gain of $G_{\mathrm{e}} /\left(Z_{\mathrm{o}}+Z_{\mathrm{h}}\right)$ without changing the system stability. This method can effectively decay the current-sharing error of power modules caused by the offset voltage.

\section{ACKNOWLEDGMENT}

This work was supported by the Program for New Century Excellent Talents in University (No.NCET-10-0367) and the Hunan Provincial Natural Science Foundation of China (No.11JJ2023)

\section{REFERENCES}

[1] K. Kondo and H. Murakami, "Crystal growth of electrolytic cu foil," J. Eeletrochem Soc, Vol. 151, No.7, pp. 514-518, 2004.

[2] L. Cheng and Q. Yongyin, "Novel high-power copper electrolytic rectifier based on auto disturbance rejection controller," International Conference on Mechatronic Science, Electric Engineering and Computer (MEC), pp. 58-61, 2011.

[3] J. R. Rodriguez, J. Pontt, C. Silva, E. P. Wiechmann, P. W. Hammond, F. W. Santucci, R. Álvarez, R. Musalem, S. Kouro, and P. Lezana, "Large current rectifiers: State of the 
art and future trends," IEEE Trans. Ind. Electron., Vol. 52, No. 3, pp.738-746, Jun. 2005

[4] B.-R. Lin and C.-C. Chen, "Analysis, design and implementation of an interleaved DC/DC converter with series-connected transformers," Journal of Power Electronics, Vol. 12, No. 4, pp. 643-653, Jul. 2012

[5] F. Ma, A. Luo, and H. Xiao, "High-power high-efficient and simplified high-frequency switching power supply for electrolytic plating," in Proc. the CSEE, Vol. 32, No. 21, pp. 71-78, Jul. 2012

[6] S.-J. Cheng, Y.-K. Lo, H.-J. Chiu, and S.-W. Kuo, "High-efficiency digital-controlled interleaved power converter for high-power PEM fuel-cell applications," IEEE Trans. Ind. Electron., Vol. 60, No. 2, pp. 773-780, Feb. 2013.

[7] D. S. Wijeratne, and G. Moschopoulos, "A three-phase single-stage AC-DC PWM buck-type full-bridge converter: Analysis, design, and characteristics," IEEE Trans. Ind. Electron., Vol. 60, No. 10, pp. 4201-4214, Oct. 2013.

[8] Y. Kawaguchi, E. Hiraki, T. Tanaka, and M. Nakaoka, "Basic study of a phase-shifted soft switching high-frequency inverter with boost PFC converter for induction heating," Journal of Power Electronics, Vol. 8, No. 2, pp.192-199, Apr. 2008.

[9] K. Raggl, T. Nussbaumer, G. Doerig, J. Biela, J. W. Kolar, "Comprehensive design and optimization of a high-power-density single-phase boost PFC," IEEE Trans. Ind. Electron., Vol. 56, No. 7, pp. 2574-2587, Jul. 2009.

[10] F. Zhang and J. Xu, "A novel PCCM boost PFC converter with fast dynamic response," IEEE Trans. Ind. Electron., Vol. 58, No. 9, pp. 4207-4216, Sep. 2011.

[11] C. Qiao and K. M. Smedley, "Three-phase unity-power-factor star-connected switch (VIENNA) rectifier with unified constant-frequency integration control," IEEE Trans. Power Electron., Vol. 18, No. 4, pp. 952-957, Jul. 2003.

[12] C. Xia, M. Wang, Z. Song, and T. Liu, "Robust model predictive current control of three-phase voltage source PWM rectifier with online disturbance observation," IEEE Trans. Ind. Informat., Vol. 8, No. 3, pp. 459-471, Aug. 2012.

[13] Z. Li, Y. Li, P. Wang, and H. Zhu, "Control of three-phase boost-type PWM rectifier in stationary frame under unbalanced input voltage," IEEE Trans. Power Electron., Vol. 25, No. 10, pp. 2521-2530, Oct. 2010.

[14] M. Beltrao de Rossiter Correa, C. B. Jacobina, E. R. C. da Silva, and A. M. N. Lima, "A general PWM strategy for four-switch three-phase inverters," IEEE Trans. Power Electron., Vol. 21, No. 6, pp. 1618-1627, Nov. 2006.

[15] J. Klima, J. Skramlik, and V. Valouch, "An analytical modeling of three-phase four-switch PWM rectifier under unbalanced supply conditions," IEEE Trans. Circuits Syst. II, Exp. Briefs, Vol. 54, No. 12, pp. 1155-1159, Dec. 2007.

[16] G. Zhu, X. Ruan, X. Wang, and Z. Yang, "Suppression of the second harmonic current and improvement of the dynamic performance for two-stage single-phase inverters," in Proc. the CSEE, Vol. 33, No. 12, pp. 72-80, Apr. 2013.

[17] J. Matas, M. Castilla, L. G. de Vicu na, J. Miret, and J. C. Vasquez, "Virtual impedance loop for droop-controlled single-phase parallel inverters using a second-order general-integrator scheme," IEEE Trans. Power Electron., Vol. 25, No. 12, pp. 2993-3001, Dec. 2010.

[18] W. Yu and D. Xu, "Control scheme of paralleled UPS system based on output virtual resistance," in Proc. the CSEE, Vol. 29, No. 24, pp. 32-39, Aug. 2009.
[19] Y. Huang and C. K. Tse, "Classification of parallel DC/DC converters part I: circuit theory," European Conference on Circuit Theory and Design, pp. 1010-1013, 2007.

[20] S. K. Mazumder, M. Tahir, and K. Acharya, "Master-slave current-sharing control of a parallel DC-DC converter system over an RF communication interface," IEEE Trans. Ind. Electron., Vol. 55, No. 1, pp. 59-66, Jan. 2008.

[21] J. J. Shieh, "Analysis and design of parallel-connected peak-current-mode-controlled switching DC/DC power supplies," IEE Proc.-Electr. Power Appl., Vol. 151, No. 4, pp. 434-442, Jul. 2004

[22] Y. Zhang, M. Yu, L. Fangrui, L. Shuming, Y. Kang, "Instantaneous current-sharing control strategy for modular UPS using virtual impedance," in Proc. the CSEE, Vol. 32, No. 21, pp. 8-14, Jul. 2012.

[23] J. M. Guerrero, J. Matas, L. García de Vicuña, M. Castilla, and J. Miret, "Decentralized control for parallel operation of distributed generation inverters using resistive output impedance," IEEE Trans. Ind. Electron., Vol. 54, No. 2, pp. 994-1004, Apr. 2007.

[24] Y.-K. Lo, C.-T. Ho, and J.-M. Wang, "Elimination of the output voltage imbalance in a half-bridge boost rectifier," IEEE Trans. Power Electron., Vol. 22, No. 4, pp. 1352-1360, Jul. 2007.

[25] C. Xia, Z. Wang, T. Shi, and X. He, "An improved control strategy of triple line-voltage cascaded voltage source converter based on proportional-resonant controller," IEEE Trans. Ind. Electron., Vol. 60, No. 7, pp. 2894-2908, Jul. 2013.

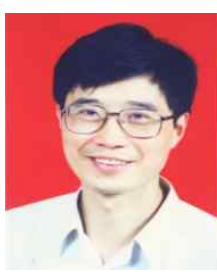

An Luo was born on July 21, 1957 in Changsha, China. He received his B.S. and M.S. degrees in 1982 and 1986 respectively from Hunan University, Changsha, China, and his Ph.D. degree in 1993 from Zhejiang University, Zhejiang, China. Between 1996 and 2002, he was a Professor at the Central South University. In 2003, he became a Professor at Hunan University, where he is currently involved in research on power conversion system, harmonics suppression and reactive power compensation, and electric power saving. He has published more than 300 journal and conference articles. $\mathrm{He}$ also serves as Chief of the Hunan Electric Science and Application Laboratory. Dr. Luo is a recipient of the 2006 National Scientific and Technological Awards of China and the 2010 National Scientific and Technological Awards of China. He currently serves as the Associate Board Chairperson of the Hunan Society for Electrical Engineering.

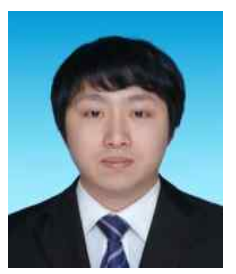

Fujun Ma was born on April 1985 in Hunan, China. He received his B.S. degree in 2004 from the College of Electrical and Information Engineering, Hunan University, Changsha, China. Since 2009, he has been working toward his Ph.D. degree in Electrical Engineering at Hunan University, Changsha, China. He is currently an assistant Professor at Hunan University. His research interests include power quality managing technique of electrified railway, electric power saving, reactive power compensation, and active power filters. 


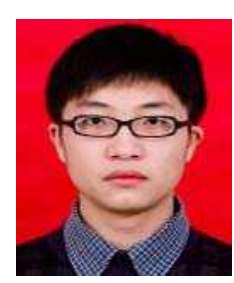

Qiaopo Xiong was born on December 3, 1988 in Nanchang, China. He received his B.S. degree in 2009 from the School of Information Science and Engineering at Central South University, Changsha, China, and his Ph.D. degree in 2014 from the College of Electrical and Information Engineering at Hunan University, Changsha, China. He is currently an Associate Research Engineer in the 722th Research Institute of CSIC, Wuhan, Hubei, China. His research interests include VSC high-voltage direct current system, static var compensator, and modular multi-level system design.

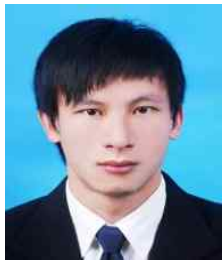

Zhixing He was born on August 12, 1989 in Hunan, China. He received his B.S. degree in 2011 from the College of Information Science and Engineering at Central South University, Changsha, China. He is currently working toward his Ph.D. degree at the College of Electrical and Information Engineering, Hunan University. His research interests include high-power industry power supply, static var compensator, and modular multi-level system design. 